\title{
Low-Complexity Detection for Index Modulation Multiple Access
}

\author{
Jun Li, Member, IEEE, Qiang Li, Shuping Dang, Member, IEEE, Miaowen Wen, Senior Member, IEEE, \\ Xue-Qin Jiang, Member, IEEE, and Yuyang Peng, Member, IEEE
}

\begin{abstract}
Index modulation multiple access (IM-MA) is recently proposed to exploit the IM concept to the uplink multiple access system, where multiple users transmit their own signals via the selected time slots. However, the computational complexity of the optimal maximum-likelihood (ML) detection in IM-MA is tremendously high when the number of users or time slots is large. In this letter, we propose a low-complexity detection method for IM-MA, which is inspired by the log likelihood ratio (LLR) algorithm. In addition, because of the heavy search burden for all LLR values, we further propose a suboptimal method to determine the permutation set, which records the number of users allocated to each time slot. Simulation results and the complexity analysis verify that the proposed detection performs closely to the optimal ML detection with reduced computational complexity.
\end{abstract}

Index Terms-Index modulation, multiple access, multi-user system, OFDM, bit error rate.

\section{INTRODUCTION}

I NDEX modulation (IM) is an efficient transmission technique, which carries the additional information bits through indices of active medias [1]-[8], where the medias can be orthogonal frequency division multiplexing (OFDM) subcarriers in the frequency domain [9], transmit or receive antennas in the spatial domain [10], spreading codes in the code domain [11], time slots or pulses in the time domain [12], [13], etc. Because only partial resource is used to convey signals and the remaining resource keeps idle, IM results in high energy efficiency (EE) and reduced inter-channel interference (ICI).

Due to the high EE and anti-interference property, IM technique has been widely used in cognitive radio (CR) networks [14], cooperative networks [15], [16], and multiple access (MA) schemes [12], [17], [19]. In [14], the inactive subcarriers of orthogonal frequency division multiplexing with index

This work was supported in part by the National Nature Science Foundation of China under Grants 61701127, 61871190 and 61671143, the Natural Science Foundation of Guangdong Province under Grant 2018B030306005, and the open research fund of National Mobile Communications Research Laboratory, Southeast University under Grant 2020D03. (Corresponding author: Miaowen Wen.)

$\mathrm{J} . \mathrm{Li}$ is with the School of Mechanical and Electrical Engineering, Guangzhou University, Guangzhou 510006, China (e-mail: lijun52018@gzhu.edu.cn).

Q. $\mathrm{Li}$ and $\mathrm{M}$. Wen are with the School of Electronic and Information Engineering, South China University of Technology, Guangzhou 510641, China, and also with the National Mobile Communications Research Laboratory, Southeast University, Nanjing 210096, China (e-mail: eeqiangli@mail.scut.edu.cn; eemwwen@scut.edu.cn).

S. Dang is with Computer, Electrical and Mathematical Science and Engineering Division, King Abdullah University of Science and Technology (KAUST), Thuwal 23955-6900, Kingdom of Saudi Arabia (e-mail: shuping.dang@kaust.edu.sa).

X.-Q. Jiang is with the School of Information Science and Technology, Donghua University, Shanghai 201620, China (e-mail: xqjiang@dhu.edu.cn).

Y. Peng is with the Faculty of Information Technology, Macau University of Science and Technology, Taipa 999078, Macau (e-mail: yypeng@must.edu.mo). modulation (OFDM-IM) are utilized to transmit the secondary user's signals in CR networks. In [15], the mapping scenario between information bits and subcarrier activation patterns (SAPs) is adaptively chosen for OFDM-IM relay networks. In order to improve the system performance, multiple relays are employed for OFDM-IM relay systems in [16]. Recently, the index modulation multiple access (IM-MA) scheme is proposed to facilitate the uplink multi-user MA system in [12], which leads to better performance than that of the conventional orthogonal MA scheme. Note that the carrier in IM-MA can also be time slot, frequency band, spread code, etc., as indicated in [17]. To enhance the spectral efficiency (SE) of IM-MA, the quadrature IM [18] is then proposed for MA in [19]. Note that different users may transmit their signals through the same carrier in IM-MA, which leads to an exhausted search when adopting the optimal maximumlikelihood (ML) detection at the base station (BS) side.

To solve the problem of the high detection complexity in IM-MA, we propose a low-complexity near-ML detection method, which is designed based on the log likelihood ratio (LLR) algorithm. Note that the transmission carrier or media for IM-MA is considered as time slots in this letter, and the proposed low-complexity detection can be easily applied to other IM-MA schemes treating the frequency band or linear block code as the transmission media. In the proposed detection, the LLR values are first calculated for all $N$ time slots and total $K$ users. Since each time slot might contain zero user, one user, and up to all $K$ users, there exist $(K+1)$ LLR values corresponding to different numbers of users for each time slot. After obtaining the LLR matrix that includes all LLR values, we aim to find the largest $K$ entries so as to generate a permutation set, which determines the number of users allocated to each time slot. However, the resulting permutation set might be illegal if the number of users corresponding to the obtained permutation set is greater than $K$. On the other hand, finding the largest $K$ values from all legal permutation set is catastrophic with a large value of $N$ or $K$. To mitigate this problem, we then propose a search algorithm to rapidly obtain the legal permutation set. The computational complexity of the proposed detection is shown to be much lower than that of the optimal ML detection. Besides, simulation results show that the proposed detection performs closely to the optimal ML detection with a negligible performance loss.

The rest of the letter is organized as follows. Section II introduces the optimal ML detection for IM-MA. The implementation and the computational complexity of the proposed detection are discussed in Section III. Simulation results are discussed in Section IV, and finally this letter is concluded in Section V.

Notations: $(\cdot)^{T}$ and $(\cdot)^{H}$ indicate the transpose and Hermi- 
tian transpose operations, respectively. $\|\cdot\|$ and $C(\cdot, \cdot)$ denote the Frobenius norm and the binomial operation of the enclosed argument, respectively. $\operatorname{diag}\{\mathbf{x}\}$ stands for a diagonal matrix whose diagonal elements are drawn from $\mathbf{x} . E\{\cdot\}$ denotes the expectation operation. The probability density function (PDF) of an enclosed random variable is denoted by $f(\cdot)$.

\section{IM-MA WITH ML DETECTION}

We consider a multi-user system with $K$ users and one BS with $N$ orthogonal time slots for uplink transmission. In IM-MA, the incoming $m=\log _{2}(N M)$ bits for each user are divided into two parts. The first part of $\log _{2} N$ bits is used to select an instant time index vector, while the second part of $\log _{2}(M)$ bits is utilized to generate the modulated symbol from the $M$-ary quadrature amplitude modulation (QAM)/phase shift keying (PSK) constellation. Note that $N$ should be a power of two for facilitating the signal transmission. The transmitted signal vector for the $k$-th user in $N$ time slots is given by

$$
\mathbf{x}_{k}=\left[0, \ldots, s_{k}, \ldots, 0\right]^{T},
$$

with

$$
\mathbf{I}_{k}=[0 \ldots, 1, \ldots, 0]^{T},
$$

where $s_{k}$ denotes the modulated symbol generated by $\log _{2}(M)$ bits, $\mathbf{I}_{k}$ represents the time index vector and the position of the non-zero element "1" in $\mathbf{I}_{k}$ is decided by the incoming $\log _{2}(N)$ bits.

For clarity and illustration purposes, we provide an example for the IM-MA scheme with $K=4, N=4$ and 4QAM ( $M=$ 4). The information bits for all users $(m=4)$ are assumed to be $[1101,0101,1011,0110]$. For user 1 with [1101], the first 2 bits [11] are used to select the 4-th time slot $\left(I_{1}=[0001]\right)$ and the last 2 bits [01] are mapped into the modulated symbol $\left(s_{1}=-1+j\right)$, which results in the transmitted signal vector $\mathbf{x}_{1}=\mathbf{I}_{1} \times s_{1}=[0,0,0,-1+j]^{T}$. Similarly, the information bits [0101] for user 2, [1011] for user 3, and [0110] for user 4 generate the transmitted signal vectors $\mathbf{x}_{2}=[0,-1+j, 0,0]^{T}$, $\mathbf{x}_{3}=[0,0,1-j, 0]^{T}$ and $\mathbf{x}_{4}=[0,-1-j, 0,0]^{T}$, respectively.

Let the $N \times 1$ vector $\mathbf{h}_{k}$ denote the block fading channel vector following the complex Gaussian distribution with zero mean and unit variance between the $k$-th user and BS. The received signal vector at the BS side is given by

$$
\mathbf{y}=\sum_{k=1}^{K} \operatorname{diag}\left\{\mathbf{h}_{k}\right\} \mathbf{x}_{k}+\mathbf{n}=\operatorname{diag}\{\mathbf{H X}\}+\mathbf{n},
$$

where $\mathbf{H}=\left[\mathbf{h}_{1}, \ldots, \mathbf{h}_{K}\right], \mathbf{X}=\left[\mathbf{x}_{1}, \ldots, \mathbf{x}_{K}\right]^{T}, \mathbf{n}$ of dimensions $N \times 1$ denotes the complex Gaussian noise with zero mean and covariance $\sigma^{2}$. The optimal ML detection at the BS is implemented as [12]

$$
\left\{\hat{\mathbf{x}}_{k}\right\}_{k=1}^{K}=\underset{s_{k} \in \mathcal{X}}{\arg \min }\left\|\mathbf{y}-\sum_{k=1}^{K} \operatorname{diag}\left\{\mathbf{h}_{k}\right\} \mathbf{x}_{k}\right\|^{2},
$$

where $\mathcal{X}$ is the candidate set containing all possible signal vectors. From (4), we can see that the optimal ML detection necessitates a holistic search over all possible candidates of $\mathbf{x}_{k}$ with $k=\{1, \ldots, K\}$ for all users, which leads to high computational complexity of $\mathcal{O}(N M)^{K}$, and becomes impractical for large values of $N, K$, and $M$.

\section{Proposed Detection Method}

In this section, we propose a low-complexity detection method, which largely reduces the computational complexity of the optimal ML detection.

\section{A. Proposed Joint LLR Detection}

Inspired by the conventional LLR detection in [9], we propose a tailored LLR detection method as follows. Since each time slot may contain $p$ users with $p \in\{0,1, \ldots, K\}$, the exact LLR value for the $n$-th time slot containing $p$ users can be calculated by

$$
\begin{aligned}
L(p, n) & =\ln \frac{\operatorname{Pr}\left(\mathrm{A}_{p, n} \mid y(n)\right)}{\operatorname{Pr}\left(\overline{\mathrm{A}}_{p, n} \mid y(n)\right)}=\ln \frac{\operatorname{Pr}\left(\mathrm{A}_{p, n}\right) f\left(y(n) \mid \mathrm{A}_{p, n}\right)}{\operatorname{Pr}\left(\overline{\mathrm{A}}_{p, n}\right) f\left(y(n) \mid \overline{\mathrm{A}}_{p, n}\right)} \\
& =\ln \frac{\operatorname{Pr}\left(\mathrm{A}_{p, n}\right) f\left(y(n) \mid \mathrm{A}_{p, n}\right)}{\sum_{i=0, i \neq p}^{K} \operatorname{Pr}\left(\mathrm{A}_{i, n}\right) f\left(y(n) \mid \mathrm{A}_{i, n}\right)}
\end{aligned}
$$

where $y(n)$ denotes the $n$-th element of $\mathbf{y}, \mathbf{A}_{p, n}$ denotes the event that $p$ users are allocated to the $n$-th time slot with the probability $\operatorname{Pr}\left(\mathrm{A}_{p, n}\right)=(1 / N)^{p}((N-1) / N)^{K-p}$, and $\overline{\mathrm{A}}_{p, n}$ represents the complementary event of $\mathrm{A}_{p, n}$. The conditional PDF $f\left(y(n) \mid \mathrm{A}_{p, n}\right)$ is shown in (6) at the top of the next page.

After calculating all LLR values, we then obtain the LLR matrix as

$$
\mathbf{L}=\left[\begin{array}{cccc}
L(0,1) & L(0,2) & \cdots & L(0, N) \\
L(1,1) & L(1,2) & \cdots & L(1, N) \\
\vdots & \vdots & \ddots & \vdots \\
L(K, 1) & L(K, 2) & \cdots & L(K, N)
\end{array}\right]
$$

It should be noted that the larger value among $\mathbf{L}$ is, the more likely the corresponding event is to happen. For instance, if $L(3,2)$ is the largest value in $\mathbf{L}$, it indicates that three users might transmit their own signals through the second time slot.

In addition, in order to figure out the user indices and the corresponding modulated symbols, we need to store the symbols and indices candidates during the calculation of each $L(p, n)$ for facilitating our proposed detection. Let $\mathbf{U}_{k}$ and $\mathbf{G}_{k}$ denote the estimated symbol matrix and user index matrix for $k$ users with $1 \leq k \leq K$, which are given by

$$
\mathbf{U}_{k}=\left[\mathbf{u}_{1}^{k}, \mathbf{u}_{2}^{k}, \ldots, \mathbf{u}_{N}^{k}\right],
$$

and

$$
\mathbf{G}_{k}=\left[\mathbf{g}_{1}^{k}, \mathbf{g}_{2}^{k}, \ldots, \mathbf{g}_{N}^{k}\right],
$$

respectively, where $\left.1 \leq u_{(k, j)}\right]^{T}$ and $\leq_{k} \quad N$, $\mathbf{u}_{j}^{k}=\left[u_{(1, j)}^{k}, u_{(2, j)}^{k}, \ldots, u_{(k, j)}^{k}\right]^{T}$ and $\mathbf{g}_{j}^{k}=$
$\left[g_{(1, j)}^{k}, g_{(2, j)}^{k}, \ldots, g_{(k, j)}^{k}\right]^{T}$.

The signal vector $\mathbf{u}_{j}^{k}$ and the corresponding index vector $\mathbf{g}_{j}^{k}$ for the $j$-th time slot are estimated by [9]

$$
\left[\mathbf{u}_{j}^{k}, \mathbf{g}_{j}^{k}\right]=\arg \min _{u_{j}^{k} \in \mathcal{X}, I_{\varphi} \in \mathcal{I}_{k}}\left|y(j)-\operatorname{diag}\left\{\mathbf{h}^{j}\left(I_{\varphi}\right)\right\} \mathbf{u}_{j}^{k}\right|^{2},
$$




$$
f\left(y(n) \mid \mathrm{A}_{p, n}\right)= \begin{cases}\frac{1}{M^{p} \pi \sigma^{2}} E\left\{\exp \left(-\frac{1}{\sigma^{2}}\left|y(n)-h_{1, n} s_{1}-\cdots-h_{p, n} s_{p}\right|^{2}\right)\right\}, & \text { if } p \neq 0 \\ \frac{1}{\pi \sigma^{2}} \exp \left(-\frac{1}{\sigma^{2}}|y(n)|^{2}\right), & \text { if } p=0\end{cases}
$$

$$
\left.\begin{array}{cccc}
t_{1} & t_{2} & t_{3} & t_{4} \\
-9.33 & -1.15 & -0.23 & -0.26 \\
-2.90 & -0.41 & -0.19 & -4.20 \\
-1.06 & -2.7 & -0.83 & -0.86 \\
-2.74 & -0.69 & -0.19 & -0.26 \\
-0.11 & 1.06 & -2.65 & -3.26
\end{array}\right]
$$

(a)

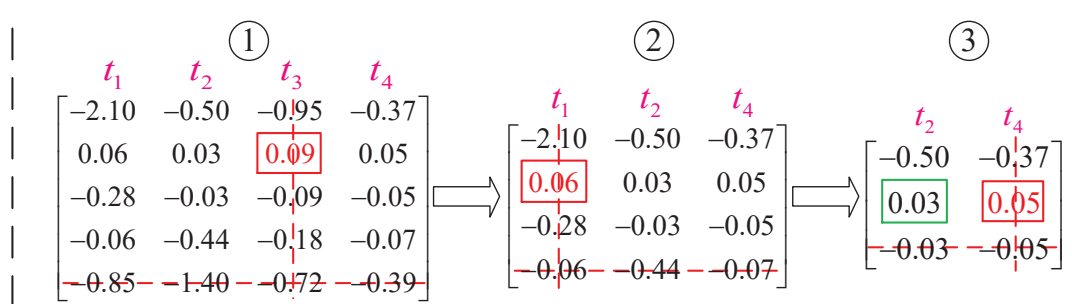

(b)

Fig. 1. An example for the proposed detection.

where $\mathcal{I}_{k}$ is the combinatorial set with a length of $C(K, k)$, $\mathbf{h}^{j}=\left[h_{1}(j), h_{2}(j), \ldots, h_{K}(j)\right]^{T}, h_{\psi}(j)$ denotes the $j$-th element of $\mathbf{h}_{\psi}$ with $1 \leq \psi \leq K$, and $\mathbf{h}^{j}\left(I_{\varphi}\right)$ contains the corresponding $I_{\varphi}$-th rows of $\mathbf{h}^{j}$ with $1 \leq \varphi \leq C(K, k)$. For better understanding, we give an example with $k=2$, $K=4, M=2$ and $N=4$. In this case, we have $\mathcal{I}_{2}=\left\{\left[\begin{array}{ll}1 & 2\end{array}\right],\left[\begin{array}{ll}1 & 3\end{array}\right], \ldots,[3 \quad 4]\right\}$ with totally $C(4,2)=6$ permutation sets. According to (10), assume that the corresponding matrices $\mathbf{U}_{2}$ and $\mathbf{G}_{2}$ are obtained by

$$
\mathbf{U}_{2}=\left[\begin{array}{llll}
+1 & -1 & +1 & +1 \\
+1 & +1 & -1 & +1
\end{array}\right]
$$

and

$$
\mathbf{G}_{2}=\left[\begin{array}{llll}
1 & 1 & 2 & 3 \\
3 & 4 & 4 & 4
\end{array}\right]
$$

From (11) and (12) with $k=2$, we can see that user 1 and user 4 probably transmit modulated symbols " -1 " and "+1" through the 2nd time slot to the BS, respectively. Besides, user 3 and user 4 probably transmit modulated symbols " +1 " and " +1 " through the fourth time slot to the BS, respectively. In a similar manner, according to $\mathbf{U}_{k}$ and $\mathbf{G}_{k}$, the corresponding modulated symbols via all time slots can be easily detected. After obtaining $\mathbf{L}, \mathbf{U}_{k}$ and $\mathbf{G}_{k}$, we can find a permutation set $\mathbf{p}$, which has the largest LLR values from all legal permutation sets in $\mathbf{L}$, and the corresponding modulated symbols and indices of time slots are straightforwardly obtained from $\mathbf{U}_{k}$ and $\mathbf{G}_{k}$. As an example with $K=N=4$, the permutation set with the largest LLR values is $\mathbf{p}=[4,0,0,0]$, which indicates that the first time slot contains all 4 users and the other time slots do not contain any user. Furthermore, the transmitted signal vector can be obtained by $\mathbf{p}, \mathbf{U}_{4}$ and $\mathbf{G}_{4}$. If the permutation set with the largest LLR values is $\mathbf{p}=[1,0,1,2]$, which indicates that the first, second, third and fourth time slots contain 1 user, 0 user, 1 user, and 2 users, respectively, and the transmitted signal vector can be retrieved based on $\mathbf{p}$, $\mathbf{U}_{1}, \mathbf{U}_{2}, \mathbf{G}_{1}$, and $\mathbf{G}_{2}$.

Note that if we perform the global search for all legal permutation sets, the proposed LLR method can achieve the same bit error rate (BER) performance as the optimal ML detection. However, the number of all legal permutation sets is huge, especially for large $K$ and $N$, which leads to a catastrophic global search among $\mathbf{L}$. Therefore, we propose an alternative search method to achieve the near-ML performance as follows. First, we initially set the permutation set as $\mathbf{p}=[0, \ldots, 0]$ and find the largest value in $\mathbf{L}$. Assume that $L(\mu, \nu)$ is the largest value in $\mathbf{L}$ with $0 \leq \mu \leq K$ and $1 \leq \nu \leq N$, which indicates that $\mu$ users transmit their symbols through the $\nu$-th time slot. The permutation set is then updated as $\mathbf{p}=[0, \ldots, \mu, \ldots, 0]$. If $\mu=K$ with $\sum_{j=1}^{N} p(j)=K$, we are led to a conclusion that all $K$ users transmit modulated symbols via the $\nu$-th time slot, and our search process is terminated. If $\mu \leq K$ with $\sum_{j=1}^{N} p(j)<K$, we find that $(K-\mu)$ users transmit their symbols through other time slots (except the $\nu$-th time slot), which should be determined by another round of search. It is straightforward that we should search the second largest value in $\mathbf{L}$. However, we may obtain an illegal permutation set, which leads to $\sum_{j=1}^{N} p(j)>K$. Therefore, before starting the next round of search, the LLR matrix $\mathbf{L}$ should be first updated by deleting the illegal elements in rows and columns of $\mathbf{L}$. After obtaining the updated $\mathbf{L}$, the above procedure is repeated until $\sum_{j=1}^{N} p(j)=K$, which finally results in the estimated permutation set. Note that the search process will determinate only when the legal permutation is obtained (i.e., $\left.\sum_{j=1}^{N} p(j)=K\right)$.

For better understanding, the search process is exemplified in Fig. 1 with $K=N=4$. Specifically, in the first step, the largest value is found to be $L(4,2)=1.06$ in $\mathbf{L}$ and the permutation set is updated as $\mathbf{p}=[0,4,0,0]$ in Fig. 1(a), which indicates that all 4 users transmit their signals through the second time slot and the search process should be stopped. In Fig. 1(b), the largest value is found to be $L(1,3)=0.09$ with the permutation set $\mathbf{p}=[0,0,1,0]$ in step 1, which indicates that one user is allocated to the third time slot $\left(\sum_{j=1}^{N} p(j)<K\right)$. In this situation, we need to keep looking for how other three users allocate their modulated symbols to the remaining (i.e., the first, second and fourth) time slots. In order to obtain a legal permutation set, the LLR matrix is updated by removing the fifth row and the third column in step 2. The largest value is consequently found to be $L(1,1)=0.06$, and the permutation set is thereby $\mathbf{p}=[1,0,1,0]$, which indicates that another one user is 


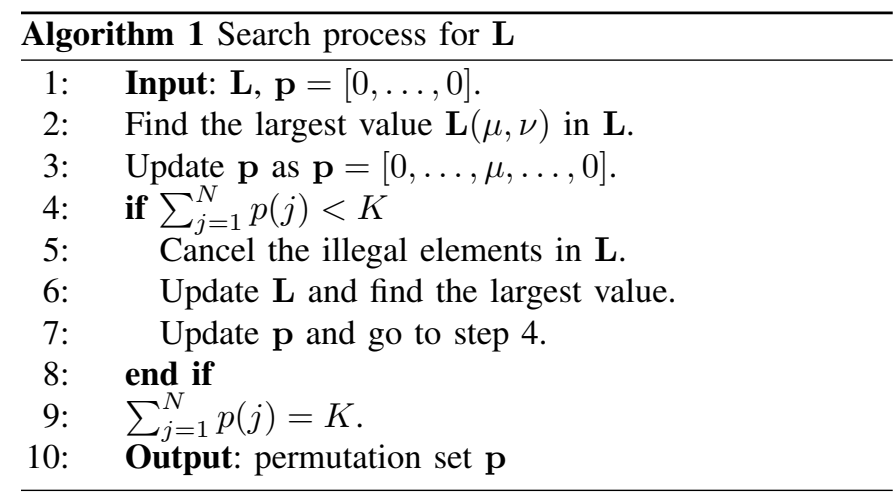

allocated to the first time slot and two users are still unknown $\left(\sum_{j=1}^{N} p(j)<K\right)$. In this case, we still need to continue the search process, and the LLR matrix is further updated in step 3, which results in the largest value $L(1,4)=0.05$ showing that one user is allocated to the fourth time slot with $\mathbf{p}=[1,0,1,1]$. To make the permutation set legal, the last one user is automatically allocated to the second time slot and the permutation set is finally obtained as $\mathbf{p}=[1,1,1,1]$. Based on the above-mentioned idea, we summarize the entire search process in Algorithm 1.

After obtaining $\mathbf{p},\left\{\mathbf{U}_{k}\right\}_{k=1}^{K}$ and $\left\{\mathbf{G}_{k}\right\}_{k=1}^{K}$, the transmitted signal vector for all users can be directly obtained and the information bits for all users are easily recovered by the demapping of the indices of active time slots and demodulation of the constellation modulated symbols.

\section{B. Complexity Analysis}

It is clear that the computational complexity of the proposed detection method only comes from the calculation of the LLR matrix $\mathbf{L}$, estimated symbol matrices $\left\{\mathbf{U}_{k}\right\}_{k=1}^{K}$, and the user index matrices $\left\{\mathbf{G}_{k}\right\}_{k=1}^{K}$. Note that we quantify the computational complexity via the number of multiplications for the proposed low-complexity detection. Accordingly, determining a single element $L(p, n)$ needs $\sum_{k=1}^{K}(k+1) M^{k} C(K, k)$ multiplications and thus identifying all elements in $\mathbf{L}$ by (7) needs $N(K+1) \sum_{k=1}^{K}(k+1) M^{k} C(K, k)$ multiplications. In addition, obtaining $\left\{\mathbf{U}_{k}\right\}_{k=1}^{K}$ and $\left\{\mathbf{G}_{k}\right\}_{k=1}^{K}$ by (10) needs $\sum_{k=1}^{K}(k+1) M^{k} C(K, k)$ multiplications. Therefore, the total number of multiplications for the proposed low-complexity detection in IM-MA is $(N K+N+1) \sum_{k=1}^{K}(k+1) M^{k} C(K, k)$. On the other hand, the total number of multiplications for the optimal ML detection in (4) is $(N+K)(N M)^{K}$. In order to clearly see the complexity reduction by adopting the proposed low-complexity detection, we present the computational complexity comparison of two detection methods in Table I. From Table I, it can be seen that the computational complexity can be greatly reduced by adopting the proposed detection method, especially for the cases with large values of $K, N$, and $M$.

\section{Upper Bound Analysis}

According to (4), the pairwise error probability (PEP) can be calculated by [12]

$$
\operatorname{Pr}(\mathbf{X} \rightarrow \hat{\mathbf{X}})=\frac{1 / 12}{\operatorname{det}\left(\mathbf{I}_{N}+q_{1} \mathbf{K A}\right)}+\frac{1 / 4}{\operatorname{det}\left(\mathbf{I}_{N}+q_{2} \mathbf{K A}\right)},
$$

TABLE I

COMPUTATIONAL COMPLEXITY COMPARISON OF THE DETECTION METHODS FOR IM-MA.

\begin{tabular}{|c||c||c||c|}
\hline$(K, N, M)$ & Proposed & ML & Reduction (\%) \\
\hline \hline$(2,4,2)$ & 260 & 384 & $32.2 \%$ \\
\hline$(4,4,2)$ & 6216 & 32768 & $81.1 \%$ \\
\hline$(4,6,4)$ & 81344 & $3 \times 10^{6}$ & $97.6 \%$ \\
\hline
\end{tabular}

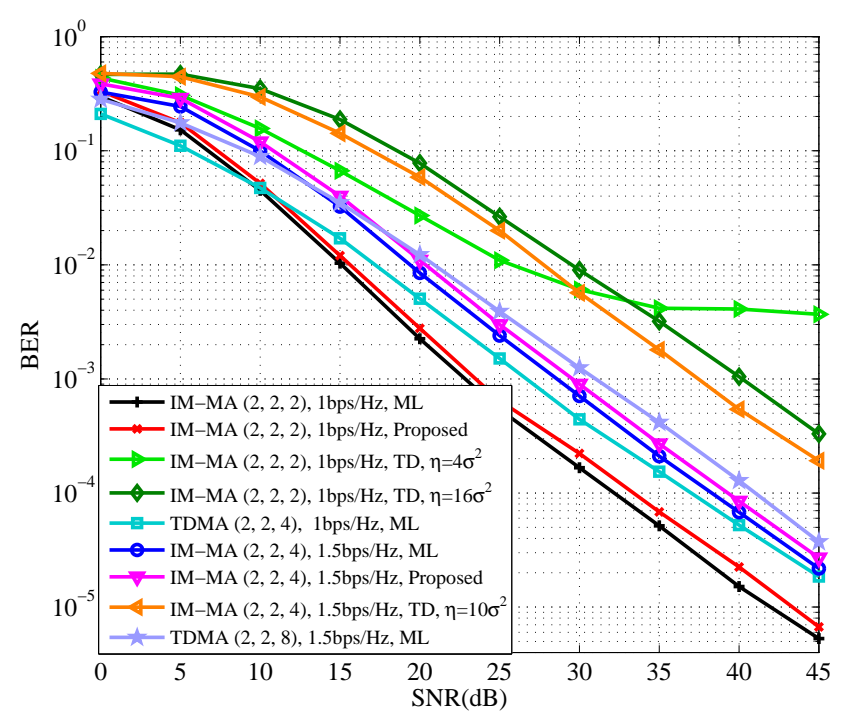

Fig. 2. Performance comparison between the proposed detection, TD and ML detection with 2 users and 2 time slots.

where $\mathbf{A}=\operatorname{diag}\{\mathbf{X}-\hat{\mathbf{X}}\}^{H} \operatorname{diag}\{\mathbf{X}-\hat{\mathbf{X}}\}, \mathbf{K}=E\left\{\mathbf{h h}^{H}\right\}$ is the covariance matrix of $\mathbf{H}, \mathbf{I}_{N}$ denotes an $N \times N$ identity matrix, $q_{1}=1 /\left(2 N_{0}\right)$ and $q_{2}=2 /\left(3 N_{0}\right)$.

After obtaining $\operatorname{Pr}(\mathbf{X} \rightarrow \hat{\mathbf{X}})$, an upper bound on BER can be derived according to the union bounding technique as

$$
P_{e} \leq \frac{1}{D 2^{D}} \sum_{\mathbf{X}} \sum_{\hat{\mathbf{X}} \neq \mathbf{X}} N(\mathbf{X} \rightarrow \hat{\mathbf{X}}) \operatorname{Pr}(\mathbf{X} \rightarrow \hat{\mathbf{X}}),
$$

where $D=K m$ and $N(\mathbf{X} \rightarrow \hat{\mathbf{X}})$ measures the number of bits in difference between $\mathbf{X}$ and $\hat{\mathbf{X}}$.

\section{Simulation Results}

In this section, we present computer simulation results for the BER performance of the optimal ML detection, the threshold detection (TD) method in [12] and the optimal ML detection for the conventional time division multiple access (TDMA). It should be noted that the magnitude of the received signal at each time slot is compared with a preset threshold $\eta$ in $\mathrm{TD}$, where no user is allocated to the corresponding time slot if the magnitude is less than $\eta$ and at least one user is allocated to the corresponding time slot if the magnitude is greater than $\eta$. For simplicity, we denote "IM-MA ( $K, N, M$ PSK/QAM)" and "TDMA ( $K, N, M$ PSK/QAM)" as the IM-MA and TDMA scheme with $K$ users and $N$ time slots with $M$-ary PSK/QAM constellations, respectively.

Fig. 2 presents the BER performance comparison results among the optimal ML detection in (4), the TD method, and 


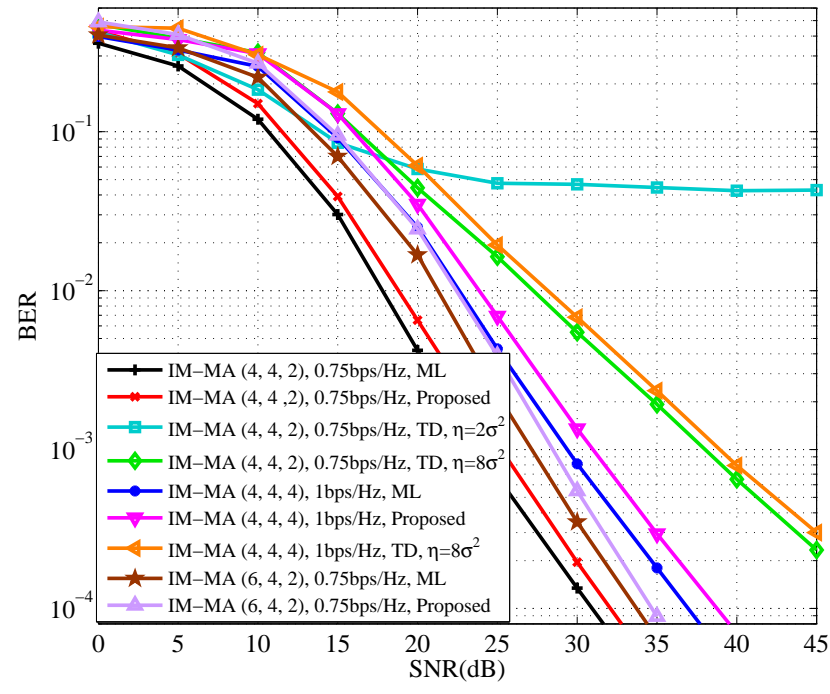

Fig. 3. Performance comparison between the proposed detection, TD and ML detection.

the proposed low-complexity detection for "IM-MA (2, 2, BPSK)" and "IM-MA (2, 2, QPSK)", and the ML detection for "TDMA (2, 2, QPSK)" and "TDMA (2, 2, 8PSK)". From Fig. 2, we can see that the proposed detection method performs closely to the optimal ML detection method with a tiny performance gap, where the performance gap comes from the abandonment of certain legal permutations during the search process. Specifically, the proposed detection results in a constant of $1 \mathrm{~dB}$ performance loss for both "IM-MA (2, 2 , BPSK)" and "IM-MA (2, 2, QPSK)" in the entire signalto-noise ratio (SNR) region. Note that due to the sensitivity of the TD method, the BER performance is quite different with different thresholds $\eta$. With $\eta=10 \sigma^{2}$ and $16 \sigma^{2}$, all curves of the TD method are far from those of the optimal ML and the proposed detections. Moreover, the TD method may result in an error floor at high SNR with an inappropriate value of $\eta$ $\left(\eta=4 \sigma^{2}\right)$. Due to the user collision impact, IM-MA is inferior to TDMA in the low SNR region. However, we can still see that the IM-MA scheme outperforms the conventional TDMA scheme with the same SE in the medium-to-high SNR region, where the SNR gain achieved by "IM-MA (2, 2, BPSK)" over "TDMA (2, 2, QPSK)" with an SE of $1 \mathrm{bps} / \mathrm{Hz} / \mathrm{user}$ is about $6 \mathrm{~dB}$, and is almost $4 \mathrm{~dB}$ by "IM-MA (2, 2, QPSK)" over "TDMA (2, 2, 8PSK)" with an SE of $1.5 \mathrm{bps} / \mathrm{Hz} / \mathrm{user}$.

Similar results can be found in Fig. 3, which present the BER performance of the optimal ML detection, the TD method, and the proposed low-complexity detection for "IMMA (4, 4, BPSK)", "IM-MA (4, 4, QPSK)" and "IM-MA $(6,4$, BPSK)". From Fig. 3, it can be seen that the proposed detection still approaches the optimal ML detection. Note that the performance gap between the proposed detection and the ML detection is about $1.5 \mathrm{~dB}$, which remains constant in the entire SNR region for both "IM-MA (4, 4, BPSK)", "IMMA (4, 4, QPSK)", and "IM-MA (6, 4, BPSK)". Besides, our proposed method is superior to the TD detection with a large performance gain. From the above results in Figs.
2 and 3 as well as Table I, it is verified that the proposed detection approaches the optimal ML detection with reduced computational complexity.

\section{CONCLUSIONS}

In this letter, we proposed the low-complexity detection to considerably reduce the computational complexity of the optimal ML detection for the IM-MA scheme. In the proposed detection, the LLR values are first calculated corresponding to all users and all time slots. Due to the high search burden of legal permutation sets among all LLR values, we further proposed the suboptimal search method to rapidly estimate the legal permutation set. Simulation results and the complexity analysis verified that the proposed detection tightly approaches the optimal ML detection with reduced computational complexity.

\section{REFERENCES}

[1] M. Wen et al., "A survey on spatial modulation in emerging wireless systems: Research progresses and applications," IEEE J. Sel. Areas Commun., vol. 37, no. 9, pp. 1949-1972, Sep. 2019.

[2] N. Ishikawa, S. Sugiura, and L. Hanzo, "50 years of permutation, spatial and index modulation: From classic RF to visible light communications and data storage," IEEE Commun. Surveys Tuts., vol. 20, no. 3, pp. 1905-1938, 3rd Quart., 2018.

[3] E. Basar, M. Wen, R. Mesleh, M. Di Renzo, Y. Xiao, and H. Haas, "Index modulation techniques for next-generation wireless networks," IEEE Access, vol. 5, pp. 16693-16746, 2017.

[4] M. Wen, X. Cheng, and L. Yang, Index Modulation for $5 G$ Wireless Communications. Berlin, Germany: Springer, 2017.

[5] T. Mao et al., "Novel index modulation techniques: A survey," IEEE Commun. Surveys Tuts., vol. 21, no. 1, pp. 315-348, 1st Quart., 2018.

[6] Q. Li, M. Wen, H. V. Poor, and F. Chen, "Information guided precoding for OFDM," IEEE Access, vol. 5, pp. 19644-19656, Nov. 2017.

[7] J. Li et al., "Layered orthogonal frequency division multiplexing with index modulation," IEEE Systems Journal, vol. 13, no. 4, pp. 3793-3802, Dec. 2019.

[8] M. Wen, X. Cheng, M. Ma, B. Jiao, and H. V. Poor, "On the achievable rate of OFDM with index modulation," IEEE Trans. Signal Process., vol. 64, no. 8, pp. 1919-1932, Apr. 2016.

[9] E. Basar, U. Aygolu, E. Panayirci, and H. V. Poor, "Orthogonal frequency division multiplexing with index modulation," IEEE Trans. Signal Process., vol. 61, no. 22, pp. 5536-5549, Nov. 2013.

[10] R. Mesleh et al., "Spatial modulation," IEEE Trans. Veh. Technol., vol. 57, no. 4, pp. 2228-2241, July 2008.

[11] Q. Li et al., "Index modulated OFDM spread spectrum," IEEE Trans. Wireless Commun., vol. 17, no. 4, pp. 2360-2374, Apr. 2018.

[12] S. Althunibat, R. Mesleh, and T. F. Rahman, "A novel uplink multiple access technique based on index-modulation concept," IEEE Trans. Commun., vol. 67, no. 7, pp. 4848-4855, July 2019.

[13] T. Ishihara et al., "Faster-than-Nyquist signaling with index modulation," IEEE Wireless Commun. Lett., vol. 6, no. 5, pp. 630-633, July 2017.

[14] J. Li, Y. Peng, Y. Yan, X.-Q. Jiang, H. Hai, and M. Zukerman, "Cognitive radio network assisted by OFDM with index modulation," IEEE Trans. Veh. Technol., vol. 69, no. 1, pp. 1106-1110, Jan. 2020.

[15] S. Dang, J. P. Coon, and G. Chen, "Adaptive OFDM with index modulation for two-hop relay-assisted networks," IEEE Trans. Wireless Commun., vol. 17, no. 3, pp. 1923-1936, Mar. 2018.

[16] J. Crawford and Y. Ko, "Cooperative OFDM-IM relay networks with partial relay selection under imperfect CSI," IEEE Trans. Veh. Technol., vol. 67, no. 10, pp. 9362-9369, Oct. 2018.

[17] S. Althunibat, R. Mesleh, and K. Qaraqe, "IM-OFDMA: A novel spectral efficient uplink multiple access based on index modulation," IEEE Trans. Veh. Technol., vol. 68, no. 10, pp. 10315-10319, Oct. 2019.

[18] B. Zheng et al., "Low-complexity ML detector and performance analysis for OFDM with in-phase/quadrature index modulation," IEEE Commun. Lett., vol. 23, no. 12, pp. 1893-1896, Nov. 2015.

[19] S. Althunibat, R. Mesleh, and K. Qaraqe, "Quadrature index modulation based multiple access scheme for 5G and beyond," IEEE Commun. Lett., vol. 23, no. 12, pp. 2257-2261, Dec. 2019. 